\title{
SPECTRAL MOMENT ESTIMATION FOR WEATHER RADARS USING A WHITENING TRANSFORMATION ON OVERSAMPLED DATA
}

\author{
Sebastián M. Torres ${ }^{(1)}$ and Dušan S. Zrnić( ${ }^{(2)}$ \\ (1) CIMMS, University of Oklahoma, Norman, OK \\ (2) National Severe Storms Laboratory, Norman, OK
}

\begin{abstract}
A method for estimation of Doppler spectral moments on pulsed weather radars is presented. This scheme operates on oversampled echoes in range; that is samples of in-phase and quadrature phase components are taken at a rate several times larger than the reciprocal of the transmitted pulse length. The aforementioned radar variables are estimated by suitably combining weighted averages of these oversampled signals in range with usual processing of samples (spaced at pulse repetition time) at a fixed range location. The weights in range are chosen such that the oversampled signals become uncorrelated and consequently the variance of estimates decreases significantly. Because estimates' errors are inversely proportional to the volume scanning times, it follows that storms can be surveyed much faster than it is possible with current processing methods, or equivalently, for the current volume scanning time, accuracy of estimates can be greatly improved.
\end{abstract}

\section{INTRODUCTION}

Weather radars provide estimates of spectral moments (i.e., signal power, mean Doppler velocity, and spectrum width) that relate to intensity of precipitation and/or refractive index fluctuations and winds. Weather signals are defined as composite echoes from a very large number of individual hydrometeors or from refractive index irregularities in clear air. These signals are sampled at discrete time delays $\tau_{s}$, where the corresponding range (or distance from the radar) is given by $r=c \tau_{s} / 2$ ( $c$ is the speed of light.) The time delay $\tau_{s}$ is also called the range time, i.e., the time it takes a transmitted pulse to make a round trip to a distance $r$. Pulses of width $\tau$ are sent every $T_{s}$ seconds, which gives origin to the sample-time, or time between samples for a fixed location in range. For each sample value there is an associated resolution volume in space with the hydrometeors that contribute the most to that sample.

The variance reduction of averaged estimates is inversely proportional to the equivalent number of independent samples $M_{I}$, which depends on the correlation between samples and the total number $M$ of samples averaged as follows [1]

$$
M_{I}=\left(\sum_{m=-M+1}^{M-1} \frac{M-|m|}{M^{2}} \rho^{2}(m)\right)^{-1},
$$

where the correlation coefficient $\rho(m)$ refers to time (from pulse to pulse) or range, and $m$ is an integer indicating the lag. For correlation in sample time, the lags are $m T_{s}$, where $T_{s}$ is the pulse repetition time; for sampling in range the lags are $m(\tau / L)$, where $\tau$ is the pulse length and $L$ a positive integer greater than one if the pulse is oversampled in range. The time correlation function depends on the Doppler spectrum width, which is one of the parameters to be estimated. If samples are averaged in range and the radar resolution volume (i.e., pulse volume) is uniformly filled with scatterers, the correlation coefficient is determined by the pulse shape and the receiver filter impulse response. After the receiver filter, the correlation coefficient of samples in range time can be determined as [1]

$\rho(m)=\left[\sum_{j=0}^{L-1} p(j) p(j-m)\right] * h(m) * h^{*}(-m)$,

where the superscript * denotes complex conjugation, $p(m)$ is the transmitted pulse shape, and $h(m)$ is the impulse response of the receiver filter. Note that the expression for the correlation coefficient along range time depends solely on parameters that are known (or can be measured) and therefore allows for its exact determination.

\section{WEATHER SIGNAL PROCESSING}

The principal purpose of radar signal processing is the accurate, efficient extraction of information from radar echoes. Modern atmospheric Doppler radars can sample an entire volume of a weather event in a short period of time. Therefore, a very large amount of data must be processed to give the user compact, comprehensible information. Note that signal processing on weather radars is primarily an estimation procedure. Target detection is not the goal of these remote sensing devices.

To obtain meaningful estimates that allow efficient quantification of weather phenomena, estimation errors must be kept below maximum allowable limits. The only parameter we can adjust to accommodate these requirements is the number of samples in the estimation process. Achieving smaller errors requires more samples, which in turn implies a slower antenna rotation rate and an overall increase in acquisition time; then probing of the weather phenomena would be performed less frequently. This statistical estimation framework becomes of particular significance if the goal is to scan a phenomenon quickly because the random-process nature of weather signals 
demands a certain amount of averaging if the desired accuracy is to be achieved. This is a trade-off in all polarimetric Doppler radar systems.

To reduce the statistical uncertainty of estimates of spectral moments it is customary to average signals from many pulses. The variance reduction of averaged estimates is inversely proportional to the equivalent number of independent samples $M_{I}$ (1), which as stated before, depends on the correlation between samples and the total number of samples averaged.

A technique that increases the number of independent samples by keeping the dwell time constant would reduce the trade-off described above. More independent samples would lower the estimates' errors at the same antenna rotation rate, or volume scans times would decrease while keeping the errors at previous levels. A well-known method to reduce the acquisition time is the pulse compression technique [2]. Pulse compression can be applied to increase the number of independent samples by averaging high-resolution estimates in range. However, most ground-based weather radars do not use pulse compression due to the need to increase the transmission bandwidth.

\section{WHITENING TRANSFORMATION}

The current implementation of spectrum moment estimators uses a simple method of averaging in range at the expense of degradation in range resolution. Simple averaging, however, does not yield the best performance when the observations are correlated.

It is know from estimation theory that classical estimators of the mean and variance of white (i.e. uncorrelated) Gaussian observations attain the Cramer-Rao lower bound. Therefore, one would like to derive a transformation on the original data based on the known correlation coefficient such that the resulting samples would be uncorrelated (or white). Still, this transformation would have to preserve the same properties that are of interest in the original sequence. Such transformation exists, and it is usually termed as "whitening" [3] or decorrelation transformation and has been applied to solve a variety of signal processing problems [4].

The procedure starts with oversampling in range so that there are $L$ samples during the pulse duration $\tau$, (that is oversampling by a factor of $L$ ). Assume that the range of depth $c \tau / 2$ is uniformly filled with scatterers. For relatively short pulses this is a common occurrence. For convenience, the contribution from the pulse volume to the sampled complex voltage $V\left(n T_{s}\right)=$ $I\left(n T_{s}\right)+j Q\left(n T_{s}\right)$ at a fixed time delay, can be decomposed into sub contributions $s(l, n)$ from $L$ contiguous slabs each $c \tau /(2 L)$ thick. The index $n$ indicates time at pulse repetition increments $T_{s}$. The voltages $s(l, n)$ are identically distributed complex Gaussian random variables, the real and imaginary parts, $\operatorname{Re}\{s(l, n)\}$ and $\operatorname{Im}\{s(l, n)\}$, have variances $\sigma^{2}$, and the power of $s(l, n)$ is $\sigma_{\mathrm{s}}^{2}=2 \sigma^{2}$. Pulse of an arbitrary shape $p(l)(l$ are time increments within the transmitted pulse which correspond to a decreasing index in range) induces weighting to the contributions from contiguous slabs such that the composite voltage is

$V(l, n)=\sum_{i=0}^{L-1} s(l+i, n) p(L-1-i)$ then the correlation of samples along range time is given by (2). For the ideal case of a rectangular pulse and infinite receiver bandwidth (2) simplifies to

$$
\rho(m)=\left\{\begin{array}{cc}
1-|m| / L & |m|<L \\
0 & \text { otherwise. }
\end{array} .\right.
$$

The procedure for implementing the whitening transformation is as follows. Define the Toeplitz symmetric correlation coefficient matrix $\mathbf{C}(\rho(0)=1$ on the main diagonal, $\rho(1)$ on the first off diagonal, $\rho(2)$ on the second off diagonal, etc.). Because this matrix is positive semidefinite, it can be decomposed into a product of a matrix $\mathbf{H}$ and its transpose as

$\mathbf{C}=\mathbf{H ~ H}^{\mathbf{t}}$

where the superscript $\mathbf{t}$ indicates matrix transpose. Any $\mathbf{H}$ that satisfies (5) is called a square root of $\mathbf{C}$ [5] and is the inverse of a whitening transformation matrix $\mathbf{W}=\mathbf{H}^{-1}$, which if applied to the range samples (in vector notation $\mathbf{V}_{\mathbf{n}}{ }^{\mathbf{t}}=[V(1, n), V(2, n), \ldots$, $V(L, n)]$ ) produces $L$ uncorrelated random variables [6]. Denote with $X(l, n)$ the sequence of time samples spaced $T_{s}$ seconds apart each of which is obtained with this procedure (in vector notation $\left.\mathbf{X}_{\mathbf{n}}{ }^{\mathbf{t}}=[X(1, n), X(2, n), \ldots, X(L, n)]\right)$, i.e.

$$
X(l, n)=\sum_{j=0}^{L-1} w_{l, j} V(j, n),
$$

where $w_{l, j}$ are the entries of the whitening transformation $\mathbf{W}$.

\subsection{Construction of the Whitening Matrix W}

In general the decomposition of $\mathbf{C}$ is not unique and many wellknown methods could be applied to generate different whitened sequences. Two prominent methods to generate whitened sequences are the eigenvalue decomposition [7] and triangular (or Gram-Schmidt orthogonalization) decomposition [7,8].

In the eigenvalue decomposition method the eigenvalues $\lambda_{i}$ of the correlation matrix $\mathbf{C}$ are computed first and $\mathbf{C}$ is represented as $\mathbf{C}=\mathbf{U} \boldsymbol{\Lambda} \mathbf{U}^{\mathbf{t}}$, where $\boldsymbol{\Lambda}$ is a diagonal matrix of eigenvalues, and $\mathbf{U}$ is the unitary transformation matrix whose columns are the eigenvectors of $\mathbf{C}$. Then, to obtain $\mathbf{W}$ a diagonal matrix $\mathbf{D}$ with elements on the diagonal equal to $\lambda_{i}^{-1 / 2}$ is constructed and $\mathbf{W}=\mathbf{H}^{-1}=\mathbf{D U}^{\mathbf{t}}$.

Triangular or Cholesky decomposition is identical to the Gram-Schmidt orthogonalization [8]. The matrix $\mathbf{H}$ is a lower triangular matrix. Hence the whitening matrix is also lower triangular. A possible advantage of triangular $\mathbf{H}$ matrices is that whitening can proceed in a pipeline manner; that is, computations can start as soon as the first sample is taken and progress through subsequent samples. Non-triangular $\mathbf{H}$ matrices require presence of all data before computations can start.

\section{SPECTRAL MOMENT ESTIMATION}

\subsection{Signal Power}

Power estimates are computed from whitened oversampled data as follows 


$$
\hat{S}=\frac{1}{M L} \sum_{l=0}^{L-1} \sum_{n=0}^{M-1}|X(l, n)|^{2}-N
$$

where $N$ is the noise power and as before, $M$ is the number of samples obtained for at a fixed range location, and $L$ is the oversampling factor. It can be proved that the improvement in variance reduction ratio when compared with the regular processing where the whitening transformation is not applied is

$$
\frac{\operatorname{Var}\left[\hat{S}_{\text {colored }}\right]}{\operatorname{Var}\left[\hat{S}_{\text {whitened }}\right]}=\frac{L^{2}+1}{2 L} .
$$

\subsection{Mean Doppler velocity and spectrum width}

Several options exist for determining the mean velocity. Autocovariance processing produces correlation estimates in sample time

$\hat{R}_{l}\left(m T_{S}\right)=\frac{1}{M} \sum_{n=0}^{M-|m|-1} X^{*}(l, n) X(l, n+m)$,

where $|m|$ is the lag index between 0 and $M-1$, and $l$ the range index between 0 and $L-1 . \quad \hat{R}_{l}\left(m T_{S}\right)$ estimates are averaged in range (over the index $l$ ) so that the variance of the estimate

$\hat{R}\left(m T_{S}\right)=\frac{1}{L} \sum_{l=0}^{L-1} \hat{R}_{l}\left(m T_{S}\right)$

decreases as $L$ increases with the same relation as in (8).

From (10) Doppler velocity and spectrum width estimates can be obtained as [1]

$$
\begin{aligned}
& \hat{v}=-\frac{\lambda}{4 \pi T_{S}} \arg \left\{\hat{R}\left(T_{S}\right)\right\}, \text { and } \\
& \hat{\sigma}_{v}=\left.\frac{\lambda}{2 \pi T_{S} \sqrt{2}}|\ln | \frac{\hat{S}}{\left|\hat{R}\left(T_{S}\right)\right|}\right|^{1 / 2} \operatorname{sgn}\left[\ln \left(\frac{\hat{S}}{\left|\hat{R}\left(T_{S}\right)\right|}\right)\right],
\end{aligned}
$$

respectively, where $\lambda$ is the transmitter wavelength.

\subsection{Performance with Additive Noise}

The presence of noise is inherent to every radar system, so it is of concern to analyze the performance of the whitening transformation under noisy conditions. When applying the whitening transformation, both signal and noise are evenly affected. The noise, which was white prior to the whitening transformation, becomes colored. It can be shown using eigenvalue decomposition of $\mathbf{C}$ that the SNR for each oversampled signal component is scaled from the original SNR by the corresponding correlation matrix eigenvalue. For $\lambda_{i}>1$, the SNR of the whitened signal increases; otherwise, the noise gets enhanced.

For a correlation matrix corresponding to (4) it is not very difficult to find a closed-form expression for the noise enhancement factor. In this case

$$
\frac{N_{\text {whitened }}}{N_{\text {colored }}}=\frac{L^{2}}{L+1} .
$$

The previous equation shows that the noise is enhanced for $L>1$ (which is always the case). Therefore, for weak SNR, the variance reduction achieved by increasing $L$ will be masked by a corresponding noise power boost. This trade-off in the presence of additive noise makes the whitening transformation useful in cases of relatively large SNR. In fact, for weather radars, the SNR of signals from storms is indeed large.

\section{RESULTS}

The proposed procedure [9] entails whitening in range the oversampled signals, processing of time samples by any one of the well-known algorithms, and combining the results from the whitened signals in range to yield significant reduction in variances of the estimates. This variance reduction occurs only if the signal-to-noise ratios are relatively high as is usually the case for most signals in weather surveillance radars. At low signal-tonoise ratios the variances increase so that there are crossover points (these are different for different estimates) of the variances. Below the cross over SNR, the classical processing produces lower variances. In general the cross over SNR depends on the variable that is to be estimated and on some other parameters (spectrum width, number of samples, etc.) An objective decision on which estimates to use, classical or the ones obtained from whitened samples in range, should be based on the SNR and possibly on estimates of other parameters that affect the variance.

Fig. 1 shows the results for the case of power estimation on colored (or correlated) and whitened noise-free observations. Theoretical results are also plotted to verify the agreement between theory and simulations. Observe that with oversampling factors of eight or more, the variance reduction is greater than four times. Fig. 2 shows the results obtained when estimating the set of three spectral moments (mean power, mean Doppler velocity, and Doppler spectrum width) in the presence of additive white Gaussian noise. This figure shows the standard deviation of estimates versus the SNR using an oversampling factor of 10 . We can see from these plots that for low SNR, the noise enhancing effect prevails, and the variance reduction obtained by the whitening transformation is masked. Beyond the crossover point, which is different for each parameter being estimated, the variance of estimates decreases as the SNR increases, until it reaches its theoretical minimum (for the ideal case of no noise). In addition it can be verified that for the range of interest $(\mathrm{SNR}>12 \mathrm{~dB})$, the estimators of spectral moments using the whitening transformation are unbiased.

\section{CONCLUSIONS}

This method allows increasing the speed of volume coverage by weather radar so that hazardous features can be timely detected. It also leads to better estimates of precipitation and wind fields. The application of this technique is possible because of two reasons:

(1) The correlation of samples in range is known exactly if the resolution volume is uniformly filled with scatterers (true 
over relatively short ranges), and the receiver bandwidth is large compared to the reciprocal of the pulse length.

(2) For all weather phenomena of interest, the SNR is relatively high, so the increase of noise power is not detrimental to the procedure.

The initial agreement between the theory and simulation results suggests that the advantages of the proposed processing will hold in practice.

\section{REFERENCES}

[1] R. J. Doviak and D. S. Zrnić, Doppler radar and weather observations, San Diego: Academic Press, 1993. 2nd ed.

[2] F. E. Nathanson, Radar Design Principles; Signal Processing and the Environment, New York: McGraw-Hill, 1969.

[3] H. L. Van Trees, Detection, Estimation, and Modulation Theory, vol. 1, New York: John Wiley \& Sons, 1968.

[4] Y. G. Sosulin and V. V. Kostrov, "Whitening filter: evolution and application," J. Comm. Technol. and Electron., vol. 43, pp. 1030-1043, 1998.

[5] D. K. Faddeev and V. N. Faddeeva, Computational Methods of Linear Algebra, San Francisco: W.H. Freeman and Company, 1963.

[6] S. M. Kay, Fundamentals of Statistical Signal Processing. Estimation Theory, Englewood Cliffs: Prentice Hall, 1993.

[7] C. W. Therrien, Discrete Random Signals and Statistical Signal Processing, Englewood Cliffs: Prentice Hall, 1992.

[8] A. Papoulis, Probability, random variables and stochastic processes, New York: McGraw-Hill, 1984.

[9] D. S. Zrnić and S. M. Torres, "Efficient estimation of spectral moments and polarimetric variables on weather radars, sonars, sodars, acoustic flow meters, and lidars," U.S. Patent Pending.
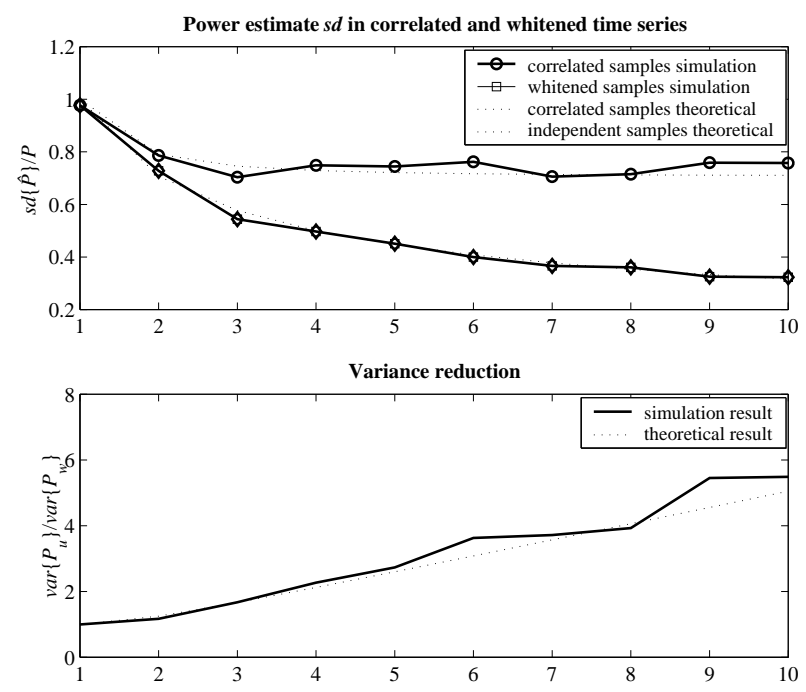

Fig. 1. Comparison of simulation results with theoretical predictions of standard deviation of power (top) and variance reduction of power estimates (bottom) vs. the oversampling factor $L$. In the bottom are the normalized mean powers.
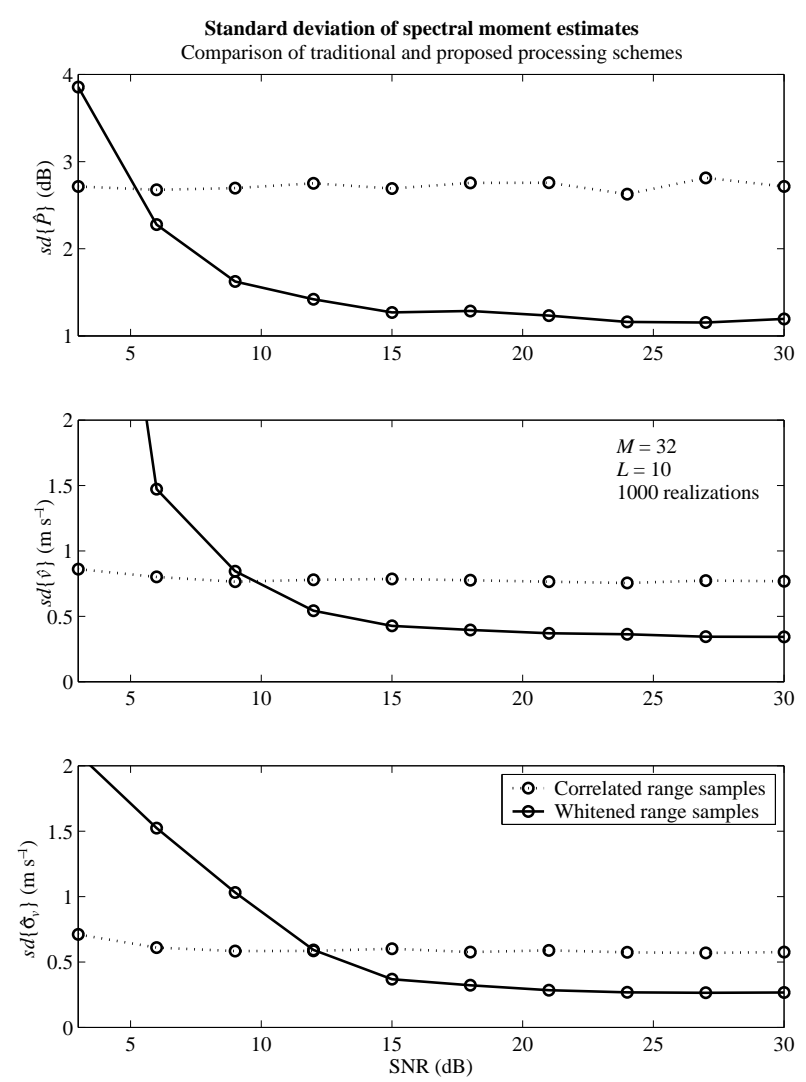

Fig. 2. Standard deviation of power (top), mean Doppler velocity (middle), and Doppler spectrum width (bottom) obtained by simulating correlated range samples and applying both traditional and proposed processing. $M$ is the number of time samples (separated by $T_{s}$ ) which are used to compute the Doppler spectrum and its moments. $L$ is the oversampling factor, i.e., the number of range samples that are used to reduce the standard error of estimates. The simulation results were obtained from 1000 realizations. For visual clarity, lines connect the simulation results (circles at SNR increments of $3 \mathrm{~dB}$ ). 